\title{
Detection of KRAS mutations using double-stranded toehold-exchange probes
}

Zhenhua $\mathrm{Wu}^{1,2}$, Tianle $\mathrm{Ma}^{3}$, Jean-Luc Coll ${ }^{4,5}$, Fangming Liu ${ }^{1}$, Honglian Zhang ${ }^{1}$, Yunfei $\mathrm{Ma}^{1}$, Zhishuo Wang ${ }^{1,2}$, Qinghui $\operatorname{Jin}^{1}$, Hongju Mao ${ }^{1 *}$, Jianlong Zhao ${ }^{1 *}$

${ }^{1}$ State Key Laboratory of Transducer Technology, Shanghai Institute of Microsystem and Information Technology, Chinese Academy of Sciences, Shanghai 200050, China.

${ }^{2}$ University of Chinese Academy of Sciences, Beijing 100039, China.

${ }^{3}$ Department of Gastroenterology, Ruijin Hospital, Shanghai Jiao Tong University School of Medicine, Shanghai 200025, China.

${ }^{4}$ INSERM U823, Institut Albert Bonniot, Grenoble, France.

${ }^{5}$ Université Joseph Fourier UJF, Grenoble, France.

*Corresponding author. Tel: +86-21-62511070-8705; Fax: +86-21-62511070-8714;

E-mail: hjmao@mail.sim.ac.cn.

*Corresponding author. Tel: +86-21-62511070-8702; Fax: +86-21-62511070-8714;

E-mail: jlzhao@mail.sim.ac.cn. 


\begin{abstract}
Detection of KRAS mutations in cancer tissues is immensely valuable for the identification of personalized genotype-based therapy. Here, we employed a double-stranded toehold-exchange probe, which is labeled with fluorescent molecules (FAM) and quenchers (Dabcyl), to detect KRAS mutations in cancer tissues. This probe was able to differentiate the intended mutation in a sample containing as little as 5\% mutant alleles in a background of wild-type DNA. This probe also performed robustly at a wide range of conditions, for examples, from $4^{\circ} \mathrm{C}$ to $37^{\circ} \mathrm{C}$, from $200 \mathrm{mM}$ $\mathrm{Na}^{+}$to $1 \mathrm{M} \mathrm{Na}^{+}$, and from $200 \mathrm{mM} \mathrm{K}$ to $500 \mathrm{mM} \mathrm{K}^{+}$. Furthermore, we validated the practicality of this probe in a clinical setting using 8 pairs of cancer tissue samples and their NT (corresponding adjacent nontumorous tissue) samples. All the results generated from the probe detection agreed with those from direct sequencing. Combining features of extreme high specificity and robustness, this probe is a valuable tool for reliable diagnosis of cancer-related mutations.
\end{abstract}

Keywords: DNA; Mutation; Toehold-exchange Probe; KRAS 


\section{Introduction}

Diagnosis of DNA mutations in cancer tissues can affect the choice of treatment for patients. For example, mutations in the KRAS gene often precede the development of malignancy (Santini et al., 2008; Voorham et al., 2013). KRAS mutations are fairly common in human cancers, such as pancreatic, colorectal and Non-Small Cell Lung cancers (Jancik et al., 2012). In addition, the presence of KRAS mutations can significantly reduce the effectiveness of certain therapies. Therefore, accurate diagnosis of KRAS mutations can help identify the appropriate personalized anticancer therapy that is best for a given patient (Jančík et al., 2010).

Various techniques have been developed for the detection of mutations, among which direct sequencing is considered as the gold standard for mutation diagnosis (Eklöf et al., 2013). However, direct sequencing relies exclusively on expensive DNA-sequencing machines and is limited by low sensitivity (at least $25 \%$ of DNA alleles in sample has to be mutated for proper detection) (Phipps et al., 2012; Ren et al., 2012). A number of new methods have emerged in recent years, like enzyme-based diagnostic assays (Botstein et al., 1980; Hall et al., 2000; Landegren et al., 1988; Tong et al., 2001), allele-specific polymerase chain reaction (PCR) (Almoguera et al., 1988), mutation specific antibodies (Yu et al., 2009) and so forth (Jancik et al., 2012; Tierling et al., 2012). All of these techniques show high sensitivity and specificity for mutation detection. Moreover, in order to meet the demand for near-patient testing, other researchers have developed convenient techniques; for example, the isothermal solid-phase amplification method integrated in a microsystem has been proven to be efficient ( $<20$ min) without involving thermal cycling (Shin et al., 2013; Shin et al., 2015). Sherry Xi Chen, et al. described a conditional double-stranded toehold-exchange probe, which is designed by precise thermodynamic calculations (Chen et al., 2013). This probe is capable of 
discriminating single-base mutations with extremely high specificity and robustness. Furthermore, it can directly detect mutations in PCR products without additional purification procedures and temperature-control equipment, which has been proven to be valuable for point-of-care testing.

In this study, we used the conditional double-stranded toehold-exchange probe to detect KRAS mutations in cancer tissues. The probe here is different from Chen's system, because it can accurately identify the type of mutations. It was able to differentiate the intended mutation in a sample containing as little as $5 \%$ mutant alleles in the background of wild-type DNA. In addition to its high sensitivity, the probe also showed robustness over a wide range of conditions (from $4^{\circ} \mathrm{C}$ to $37^{\circ} \mathrm{C}$, from $200 \mathrm{mM} \mathrm{Na}^{+}$to $1 \mathrm{M} \mathrm{Na}^{+}$, from $200 \mathrm{mM} \mathrm{K}^{+}$to $500 \mathrm{mM} \mathrm{K}{ }^{+}$). In order to validate the probe for practical use, 8 pairs of cancer tissue samples and their NT (corresponding adjacent nontumorous tissue) samples were examined using this probe. These samples were composed of 6 mutant samples of two types of single-base mutations and 2 wild-type samples. They were selected from 12 pairs of colorectal cancer tissue / NT samples and 52 pairs of lung adenocarcinoma / NT samples via direct sequencing. All of the diagnostic results of the probe were consistent with those from DNA sequencing. With its high specificity and robustness, our study developed a reliable assay for KRAS mutation diagnosis in cancer tissues.

\section{Material and methods}

\subsection{Oligonucleotide Synthesis and Storage Conditions}

The Fluorophore- and quencher-labeled oligonucleotides were synthesized and purified by high-performance liquid chromatography (HPLC) by Takara Bio (Dalian, China). Other unmodified oligonucleotides were purchased as primers from Sangon biotech (Shanghai, China) and purified by 
polyacrylamide gel electrophoresis (PAGE). The strands used to construct the probe were resuspended separately at a concentration of $100 \mu \mathrm{M}$ and stored in Tris-EDTA (TE) buffer (10 mM Tris- $\mathrm{HCl}, \mathrm{pH}=8$ with $1 \mathrm{mM}$ EDTA) at $-20^{\circ} \mathrm{C}$. The primers used in the $\mathrm{PCR}$ procedures were resuspended at a concentration of $100 \mu \mathrm{M}$ in Milli-Q water from a Millipore purification system and stored at $-20^{\circ} \mathrm{C}$. The sequences of the oligonucleotides are listed in Supplementary Material, Table S-1.

\subsection{Probe Design and Calculation Of $\Delta \mathrm{G}^{\circ}$}

The probe was designed under the theoretical framework presented by Zhang et al. (Zhang et al., 2012) and Chen et al. (Chen et al., 2013). 6-nt-long initiation toehold and 4-nt-long balancing toehold were used in this study. The different lengths of the toeholds were aimed to balance the extra free energy resulted from fluorophore-quencher contact (Marras et al., 2002) and to ensure that the reaction free energy $\Delta \mathrm{G}^{\circ} \approx 0 \mathrm{kcal} / \mathrm{mol}$. DINAMelt Web Server (Markham and Zuker, 2005) was used to calculate the standard free energy $\Delta \mathrm{G}^{\circ}$ of each complex. For the calculations, the salt concentrations were set to $500 \mathrm{mM} \mathrm{Na}^{+}$and $0 \mathrm{M} \mathrm{Mg}^{2+}$. The temperature was set to $25^{\circ} \mathrm{C}$.

\subsection{Probe Preparation}

The probe consists of four different strands. Two of them were labeled with a fluorescent molecule (FAM) and a quencher (Dabcyl), respectively. The other two were unlabeled strands. Prior to the experiments, the strands were diluted to $1 \mu \mathrm{M}$ with Tris-EDTA (TE) buffer $(10 \mathrm{mM}$ Tris-HCl, $\mathrm{pH}=8$ with $1 \mathrm{mM}$ EDTA). They were then mixed stoichiometrically, during which TE buffer containing $4 \mathrm{M} \mathrm{NaCl}$ was added to bring the final $\mathrm{Na}^{+}$concentration to $500 \mathrm{mM}$. The final probe 
concentration was $200 \mathrm{nM}$. Finally, the mixture was thermally annealed from $98^{\circ} \mathrm{C}$ to $25^{\circ} \mathrm{C}$ over 73 minutes.

\subsection{DNA Extraction}

In this study, the wild-type and mutant KRAS DNA templates were prepared by overlap extension PCR and cloned by pUC-T Quick Ligation Kit (CWBIO, Beijing, China) according to the manufacturer's instructions. Both templates were diluted to $10^{5}$ copies/ $\mu \mathrm{L}$. Subsequently, we prepared serial mixtures containing $50 \%, 25 \%, 10 \%, 5 \%$, and $1 \%$ mutant templates within a background of wild type template. Lung advenocarcinoma tissue samples, colorectal cancers tissue samples and their corresponding adjacent nontumorous tissue (NT) samples were obtained from patients who underwent primary surgical resection of cancer tissues at Shanghai Ruijin Hospital. Samples were obtained with informed consent. They were stored separately at $-80^{\circ} \mathrm{C}$. Genomic DNA was extracted from the frozen tissue samples using AllPrep DNA/RNA Mini Kit (50) (QIAGEN) according to the manufacturer's instructions. All DNA samples were stored at $-80^{\circ} \mathrm{C}$ until use.

\subsection{Target Preparation}

KRAS subsequences amplified from the DNA templates were constructed through several PCR and annealing procedures. Since KRAS mutations primarily occur at codons 12 or 13 of exon 2, all of the PCR primers were designed to amplify the corresponding region. The first PCR process consists of a thermal denaturation step at $95^{\circ} \mathrm{C}$ for $10 \mathrm{~min}$; followed by 35 cycles of $95^{\circ} \mathrm{C}$ for $10 \mathrm{~s}$, $58^{\circ} \mathrm{C}$ for $10 \mathrm{~s}$, and $72^{\circ} \mathrm{C}$ for $30 \mathrm{~s}$; a final extension step at $72^{\circ} \mathrm{C}$ for $7 \mathrm{~min}$; and then preservation at $4^{\circ} \mathrm{C} .2 \mu \mathrm{L}$ of DNA templates (or $50 \mathrm{ng}$ Genomic DNAs from the tissue samples) were amplified in a 
total volume of $20 \mu \mathrm{L}$ reaction containing $1 \times$ PCR buffer (Takara Bio, Dalian, China), $0.25 \mathrm{mM}$ deoxynucleotide triphosphate, $2.5 \mathrm{mM} \mathrm{MgCl}$, 2 units of Taq DNA polymerase (Takara Bio, Dalian, China), and $250 \mathrm{nM}$ of each primer (Primers F and R listed in Supplementary Material, Table S-1).

The PCR products from the above procedures were used as templates for a subsequent unbalanced PCR procedure. This procedure started with a thermal denaturation step at $95^{\circ} \mathrm{C}$ for 10 min; 30 cycles of $95^{\circ} \mathrm{C}$ for $10 \mathrm{~s}, 58^{\circ} \mathrm{C}$ for $10 \mathrm{~s}$, and $72^{\circ} \mathrm{C}$ for $30 \mathrm{~s}$; and then preservation at $4^{\circ} \mathrm{C}$. Through this PCR procedure, the two strands of the target were generated separately, with $500 \mathrm{nM}$ primer $\mathrm{F} 1,10 \mathrm{nM}$ primer $\mathrm{R} 1$ for one strand and $500 \mathrm{nM}$ primer $\mathrm{R} 2,10 \mathrm{nM}$ primer $\mathrm{F} 1$ for the other. (Primers F1, R1 and R2 are listed in Supplementary Material, Table S-1) The two strands amplified by unbalanced PCR were directly mixed. Meanwhile, aqueous solution containing $4 \mathrm{M} \mathrm{NaCl}$ was added to the mixture to achieve a final $\mathrm{Na}^{+}$concentration of $500 \mathrm{mM}$. Finally, the mixture was uniformly cooled from $98^{\circ} \mathrm{C}$ to $25^{\circ} \mathrm{C}$ over $73 \mathrm{~min}$.

\subsection{Direct Sequencing}

The PCR process was the same as the first PCR procedure described in the Target Preparation section. Direct sequencing of the PCR products was performed by Sangon biotech (Shanghai, China). In this experiment, all the DNA templates, and 12 pairs of colorectal cancer/NT samples and 52 pairs of lung adenocarcinoma/NT samples were subjected to direct sequencing.

\subsection{Detection of KRAS Mutations}

Target and probe were directly mixed at a ratio of 3:1 and stored at room temperature for 10 hours to achieve equilibration (the initial concentration of the probe was $50 \mathrm{nM}$ ). Fluorescence was 
observed by a fluorescence microscope (BX51, OLYMPUS) equipped with a $100 \mathrm{~W}$ mercury lamp, a $2 \times$ objective lens, a CCD camera and a filter. The images were captured by the CCD camera. A Horiba FluoroMax-4 spectrofluorimeter and Orient KOJI Semi-Micro spectrofluorometer cuvettes were utilized to perform Emission fluorescence measurements. Slit sizes were set at $1.5 \mathrm{~nm}$ for all monochromators. All processes were conducted at room temperature $\left(25 \pm 1^{\circ} \mathrm{C}\right)$. For this experiment, all probes were modified with the FAM fluorophores (excitation $492 \mathrm{~nm}$, emission $518 \mathrm{~nm}$ ).

\section{Results and Discussion}

\subsection{Principle of KRAS Mutation Detection}

\section{Insert Fig. 1}

The key step of the detection process is a double-stranded displacement reaction. Through this reaction, the probe can discriminate the intended mutation from others. With the designed toeholds on the 5' end of the unbalanced PCR primers, the target forms forked toeholds at one end of the sequence. As shown in Fig. 1, the target is directly mixed with the probe. The toeholds of the same color are complementary, and hybridize to each other in order to initiate the process of double-stranded displacement reaction. The reactants undergo a branch migration process to exchange one strand with each other, and finally release the two products. Then the FAM and quencher detach from each other. The fluorescent intensity reflects the yield of the product.

The yield of the reaction products mainly depends on the reaction free energy $\Delta \mathrm{G}^{\circ}$. On one 
hand, we designed the sequences of the toeholds so that the reaction involving the intended mutant target has $\Delta \mathrm{G}^{\circ} \mathrm{m} \approx 0 \mathrm{kcal} / \mathrm{mol}$. This ensures a rational yield of fluorescence signal. On the other hand, reaction involving the wild-type target will form 2 mismatch bubbles and will have $\Delta \mathrm{G}^{\circ}{ }_{\mathrm{w}} \approx+8$ $\mathrm{kcal} / \mathrm{mol}$. This positive free energy will severely reduce the final yield of the fluorescence (Chen et al., 2013). This particular design allows the probe to discriminate the intended mutant target from others and effectively diagnose KRAS single-base mutations.

\subsection{Detection of KRAS Single-base Mutation at Codon 12}

\section{Insert Fig. 2}

The probe was tested on the indicated DNA templates and was observed to display high specificity for distinguishing the intended mutation. Fig. 2a shows that the intended mutant target (Mut-1) emitted stronger response compared to the wild-type target. The background curve represents the background fluorescence generated by the probe mixed with buffer only. Specificity can be quantitated as discrimination factor $Q=\left(I_{M}-I_{B}\right) /\left(I_{W}-I_{B}\right)$, where $I_{M}, I_{W}$ and $I_{B}$ are the fluorescent intensities (wavelength $=518 \mathrm{~nm}$ ) of the mutant target, the wild-type target, and the background, respectively (Zhang et al., 2012). As shown in Fig. 2a, the value of $Q$ was 50.8, indicating that signals of mutant target showed roughly 50-fold excess compared to that of wild-type target. Fig. 2b shows the direct sequencing result of the targets, which is consistent with our detection results. Our data demonstrates that this probe is capable of discriminating the intended mutant KRAS subsequence from wild-type sequences. 


\subsection{Robustness}

At a typical clinical setting, convenient techniques that require no extra temperature-control equipment or purification procedure are in high demand. In order for our probe to be applicable for near-patient testing, the probe should operate robustly over a wide range of conditions. In other words, neither temperature nor salinity should affect its specificity. In fact, our results show that this probe is effective under various conditions (from $4^{\circ} \mathrm{C}$ to $37^{\circ} \mathrm{C}$, from $200 \mathrm{mM} \mathrm{Na}$ to $1 \mathrm{M} \mathrm{Na}^{+}$, and from $200 \mathrm{mM} \mathrm{K}^{+}$to $500 \mathrm{mM} \mathrm{K}$ ).

\section{Insert Fig. 3}

To validate the robustness of the probe, we performed the detection procedure in different salinity conditions (Fig. 3). The targets were generated from the indicated DNA templates; meanwhile, aqueous solution containing $4 \mathrm{M} \mathrm{NaCl}$ or $\mathrm{KCl}$ was added to the target to create the various concentrations of $\mathrm{Na}^{+}$or $\mathrm{K}^{+}$. Then, the targets were directly mixed with the probes under these conditions, respectively. Using the result at the $500 \mathrm{mM} \mathrm{Na}{ }^{+}$condition as an example (Fig. 3b), the emission spectra of the wild-type target and the background were almost the same, suggesting that the wild-type target generated almost no fluorescent response. In contrast, the fluorescent response from the mutant target was obvious, and the value of discrimination factor $Q$ is 50.8 . Our results demonstrated that the probe is capable of achieving extremely high specificity at $\mathrm{Na}^{+}$ concentration of $500 \mathrm{mM}$. As for other cation conditions shown in Fig. 3, although the probe specificity at $200 \mathrm{mM} \mathrm{Na}{ }^{+}(Q=18.5)$ was a little lower than at other conditions, the signal of mutant target still showed roughly 18 -fold excess compared to that of wild-type target. The overall result 
still exhibited high robustness.

\section{Insert Fig. 4}

We also performed the detection procedure at different temperatures (ranges from $4{ }^{\circ} \mathrm{C}$ to $37^{\circ} \mathrm{C}$ ). (Fig. 4) The concentration of $\mathrm{Na}^{+}$was $500 \mathrm{mM}$ and the temperature was controlled by a temperature bath. Using the results at $25^{\circ} \mathrm{C}$ as an example, the signal of mutant target showed roughly 50-fold excess compared to that of wild-type target $(Q=50.8)$. Similar results were observed at $4^{\circ} \mathrm{C}$. Although the fluorescence of the mutant target was slightly weaker than that at $25^{\circ} \mathrm{C}$, our probe still maintained high specificity overall $(Q=44.6)$. When the temperature was increased to $37^{\circ} \mathrm{C}$, signals from both the mutant and the wild-type target increased, however, the signal of mutant target still showed roughly 15-fold excess compared to that of wild-type target $(Q=$ 15.3). Therefore, we concluded that our probe performs robustly over a wide range of temperatures from $4^{\circ} \mathrm{C}$ to $37^{\circ} \mathrm{C}$.

From a thermodynamic standpoint, changing the salinity and temperature of the reaction should affect the thermodynamic properties of the reactants and the products in the detection procedure. These potential effects were canceled out due to the deliberate probe design, which ensures that $\Delta \mathrm{G}_{\mathrm{m}}^{\circ} \approx 0 \mathrm{kcal} / \mathrm{mol}$. As a result, the high specificity of our probe was preserved (Chen et al., 2013).

\subsection{Sensitivity of KRAS mutation detection}

\section{Insert Fig. 5}


In practice, the mutant alleles are always present in populations containing numerous wild-type DNA. Thus, it is important to test the probe in the presence of wild-type template. As shown in Fig. 5a, we used the indicated mixtures to determine the sensitivity of this probe. These serial mixtures contained $50 \%, 25 \%, 10 \%, 5 \%, 1 \%$ mutant templates, and the $0 \%$ contained wild-type templates only. Although the mutant alleles were not detected in the mixture containing $1 \%$ mutant templates, they were easily detected in mixture containing 5\% mutant templates. Moreover, there was a linear range over the $5 \%$ to $50 \%$ range $\left(\mathrm{R}^{2}=0.9934\right)$ in this detection, and the detection limit was about 5\% (based on $\mathrm{S} / \mathrm{N}=3$ ) (Fig. 5b). However, the direct sequencing was only able to identify the mutant templates when the percentage of them is $>25 \%$ (Fig. 5c). Therefore, this probe could be used to develop detection schemes for clinical use.

\subsection{Diagnosis of KRAS Mutations in Patient Samples}

\section{Insert Fig. 6}

Finally, we validated the clinical usability of the probe using 8 pairs of cancer / NT samples collected from patients. All the experimental results agree with those generated by direct sequencing. These samples were selected from 12 colorectal cancer tissue samples and 52 lung adenocarcinoma samples that were subjected to direct sequencing. Fig. 6a shows the sequencing results of these 8 samples numbered from 1 to 8 . In these samples, 2 of colorectal cancer tissues (sample 1 , 2) were found to carry a mutation in codon 12 of the KRAS gene (Gly > Asp), denoted as Mut-1. Four of the 
lung adenocarcinoma tissues (sample 3-6) were found to carry a mutation in codon 12 (Gly > Cys), denoted as Mut-2. The other 2 samples (sample 7, 8) were wild type. Meanwhile, no mutation was found in their NT samples. Two probes were designed for the two types of mutation, respectively (Supplementary Material, Table S-1). As shown in Fig. 6b, a probe was designed for Mut-1 and was used to examine all 8 pairs of the samples. Only cancer tissue sample 1 and 2 emitted strong fluorescence, while other cancer tissues generate little to no response. Similarly, as shown in Fig. 6c, strong fluorescent responses were only observed in cancer tissue sample 3-6 when treated with a probe designed for Mut-2. All of our experimental results confirm those generated from direct sequencing. Therefore, our probe is reliable and practical for the identification of the mutant status of KRAS subsequences.

\section{Conclusions}

We reported a reliable probe to identify single-base mutations in the KRAS gene. This probe shows extremely high specificity and robustness in various temperature and salinity conditions, which promotes its usability in the clinical setting. In addition, the successful detection of patient samples validated the clinical utility of this probe. Not only is this probe performs as well as direct sequencing in identifying point mutations, it is also more convenient. Although the mutations were observed by fluorescence in this experiment, the probe can be combined with other techniques, for examples, other electrochemical methods and semiconductor devices, which are also commonly used as DNA sensors. In summary, we have demonstrated a highly specific and effective single-base mutation detection probe. This mutation diagnosis probe is especially valuable due to its clinical utility and will contribute immensely to improving personalized genotype-based therapies. 


\section{Acknowledgment}

This work was supported by Grants from the National Basic Research Program of China (973

Program) (No. 2012CB933303), the National Science Foundation of China (No. 61571429, 61401442, 81101404, 21275153, 61271162, 61571428 and 61571077), Shanghai Clinical Center/Shanghai Xuhui Central Hospital, Chinese Academic of Sciences (No.BRC2012002).

\section{References}

Almoguera, C., Shibata, D., Forrester, K., Martin, J., Arnheim, N., Perucho, M., 1988. Cell 53, 549-554.

Botstein, D., White, R.L., Skolnick, M., Davis, R.W., 1980. Am. J. Hum. Genet. 32, 314-331.

Chen, S.X., Zhang, D.Y., Seelig, G., 2013. Nat. Chem. 5, 782-789.

Eklöf, V., Wikberg, M.L., Edin, S., Dahlin, A., Jonsson, B.-A., Öberg, Å., Rutegård, J., Palmqvist, R., 2013. Br. J. Cancer 108, 2153-2163.

Hall, J.G., Eis, P.S., Law, S.M., Reynaldo, L.P., Prudent, J.R., Marshall, D.J., Allawi, H.T., Mast, A.L., Dahlberg, J.E., Kwiatkowski, R.W., 2000. Proc. Natl. Acad. Sci. U. S. A. 97, 8272-8277.

Jančík, S., Drábek, J., Radzioch, D., Hajdúch, M., 2010. Biomed Res. Int. 2010, 1-13.

Jancik, S., Drabek, J., Berkovcova, J., Xu, Y.Z., Stankova, M., Klein, J., Kolek, V., Skarda, J., Tichy, T., Grygarkova, I., 2012. J. Exp. Clin. Cancer Res. 31, 79.

Landegren, U., Kaiser, R., Sanders, J., Hood, L., 1988. Science 241, 1077-1080.

Markham, N.R., Zuker, M., 2005. Nucleic Acids Res. 33, W577-W581.

Marras, S.A., Kramer, F.R., Tyagi, S., 2002. Nucleic Acids Res. 30, e122-e122.

Phipps, A.I., Buchanan, D.D., Makar, K.W., Burnett-Hartman, A.N., Coghill, A.E., Passarelli, M.N., Baron, J.A., Ahnen, D.J., Win, A.K., Potter, J.D., 2012. Cancer Epidem. Biomar. 21, 1792-1798.

Ren, J., Li, G., Ge, J., Li, X., Zhao, Y., 2012. Dis. Colon Rectum 55, 913-923.

Santini, D., Loupakis, F., Vincenzi, B., Floriani, I., Stasi, I., Canestrari, E., Rulli, E., Maltese, P.E., Andreoni, F., Masi, G., 2008. Oncologist 13, 1270-1275.

Shin, Y., Perera, A.P., Park, M.K., 2013. Sensor. Actuat. B: Chem. 178, 200-206.

Shin, Y., Soo, R.A., Yoon, J., Perera, A.P., Yoon, Y.-J., Park, M.K., 2015. Biosens. Bioelectron. 68, 107-114. 
Tierling, S., Sers, C., Lehmann, A., Walter, J., 2012. Int. J. Cancer 130, 567-574.

Tong, A.K., Li, Z., Jones, G.S., Russo, J.J., Ju, J., 2001. Nat. Biotechnol. 19, 756-759.

Voorham, Q.J., Rondagh, E.J., Knol, D.L., van Engeland, M., Carvalho, B., Meijer, G.A., Sanduleanu, S., 2013. Am. J. Gastroenterol. 108, 1042-1056.

Yu, J., Kane, S., Wu, J., Benedettini, E., Li, D., Reeves, C., Innocenti, G., Wetzel, R., Crosby, K., Becker, A., 2009. Clin. Cancer Res. 15, 3023-3028.

Zhang, D.Y., Chen, S.X., Yin, P., 2012. Nat. Chem. 4, 208-214. 


\section{Captions}

Fig. 1. Schematic representation of the principle of the conditional double-stranded toehold-exchange probe. The target was directly mixed with the probe. The intended mutant target emitted stronger fluorescence compared to the wild-type target.

Fig. 2. Different responses of the probe to intended mutation versus wild-type sequences. (a) Comparison of the fluorescent response of the single-base mutant target and the wild-type target. The background curve represents the background fluorescence generated by the probe mixed with buffer only. The fluorescence intensities were measured by Horiba FluoroMax-4 spectrofluorometer (excitation at $493 \mathrm{~nm}$, emission at $518 \mathrm{~nm}$ ). Q was the average value of at least 3 replicates of this experiment (standard deviation=10.8). The concentration of $\mathrm{Na}^{+}$was $500 \mathrm{mM}$. The experiments were conducted at room temperature $\left(25^{\circ} \mathrm{C}\right)$. (b) Sequencing results of the targets. Wild: wild type-(12Gly-GGT). Mut-1: mutant-(12Asp-GAT).

Fig. 3. The probe is capable of discriminating single-base mutations robustly in buffer containing (a) $200 \mathrm{mM} \mathrm{Na}^{+}$, (b) $500 \mathrm{mM} \mathrm{Na}^{+}$, (c) $1 \mathrm{M} \mathrm{Na}^{+}$, (d) $200 \mathrm{mM} \mathrm{K}^{+}$, and (e) $500 \mathrm{mM} \mathrm{K}$. Q was the average value of at least 3 replicates of each experiment. (f) Q values in different salinity conditions. Error bars are the standard deviations based on at least 3 replicates of each experiment. The emission spectra were measured by Horiba FluoroMax-4 spectrofluorometer (excitation at $493 \mathrm{~nm}$, emission at $518 \mathrm{~nm})$. The experiments were conducted at room temperature $\left(25^{\circ} \mathrm{C}\right)$. 
Fig. 4. The probe discriminates single-base mutations robustly at (a) $4^{\circ} \mathrm{C}$, (b) $25^{\circ} \mathrm{C}$, and (c) $37^{\circ} \mathrm{C}$. Q was the average value of at least 3 replicates of each experiment. (d) Q values in different temperature conditions. Error bars are the standard deviations based on at least 3 replicates of each experiment. The concentration of $\mathrm{Na}^{+}$was $500 \mathrm{mM}$.

Fig. 5. Detection of KRAS mutation with serial mixtures of mutant templates and wild-type templates. The concentration of $\mathrm{Na}^{+}$was $500 \mathrm{mM}$. The experiments were conducted at room temperature $\left(25^{\circ} \mathrm{C}\right)$. The percentages of mutant templates were $50 \%, 25 \%, 10 \%, 5 \%, 1 \%$ and $0 \%$. (a) Emission spectra of the fluorescent response to the mixed templates of different ratios. (b) Fluorescence intensity (emission at $518 \mathrm{~nm}$ ) vs. percentages of mutant templates. Error bars are the standard deviation based on at least 3 replicates of each experiment. (c) Sequencing results of the targets with different percentages of mutant templates.

Fig. 6. (a) Sequencing results of the 8 pairs of patient cancer/NT samples. Sample 1, 2 are colorectal cancer tissues, and sample 3-8 are lung adenocarcinoma tissues. (b) Detection of the Mut-1. "Control" represents the fluorescent intensity generated by wild-type templates. (c) Detection of Mut-2. 

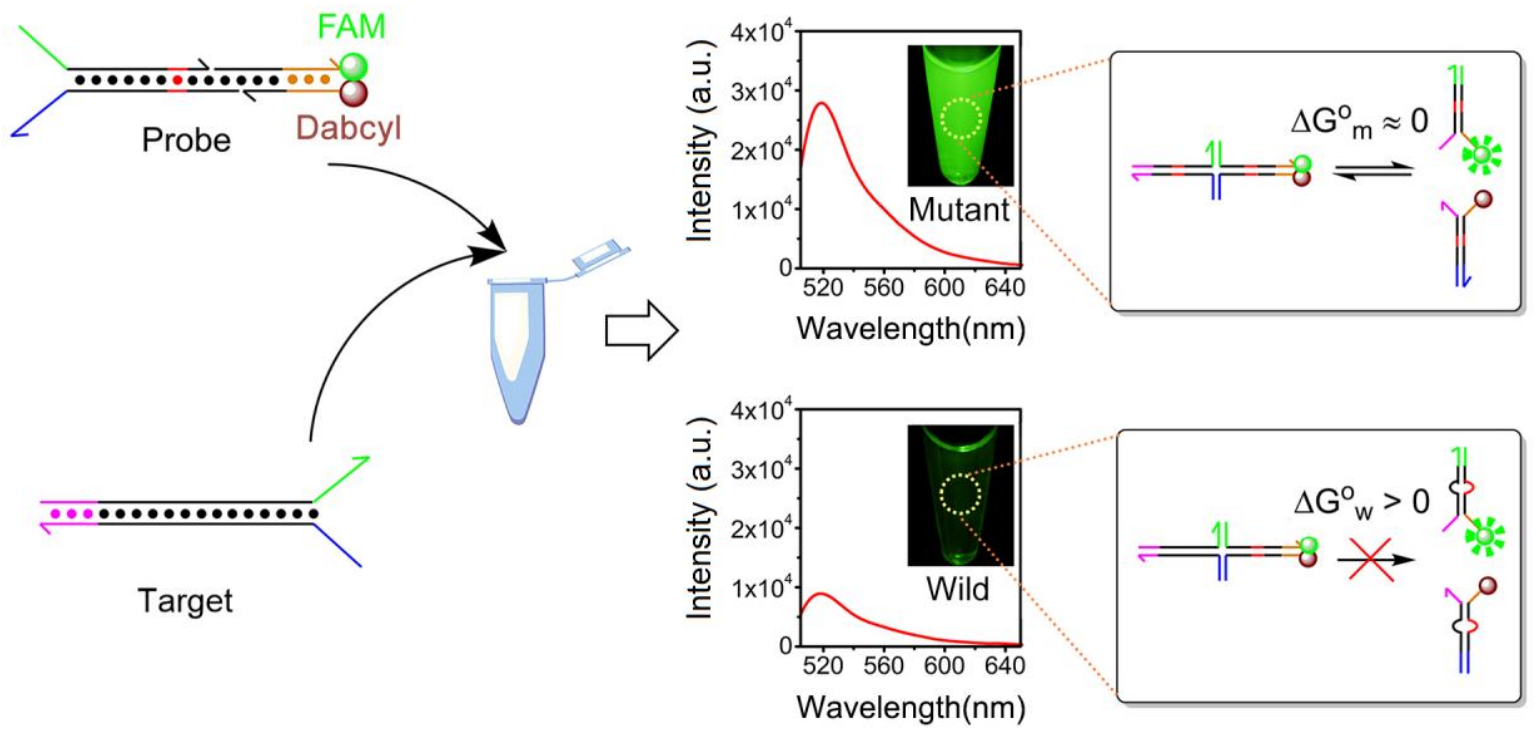

Fig. 1. Schematic representation of the principle of the conditional double-stranded toehold-exchange probe. The target was directly mixed with the probe. The intended mutant target emitted stronger fluorescence compared to the wild-type target. 

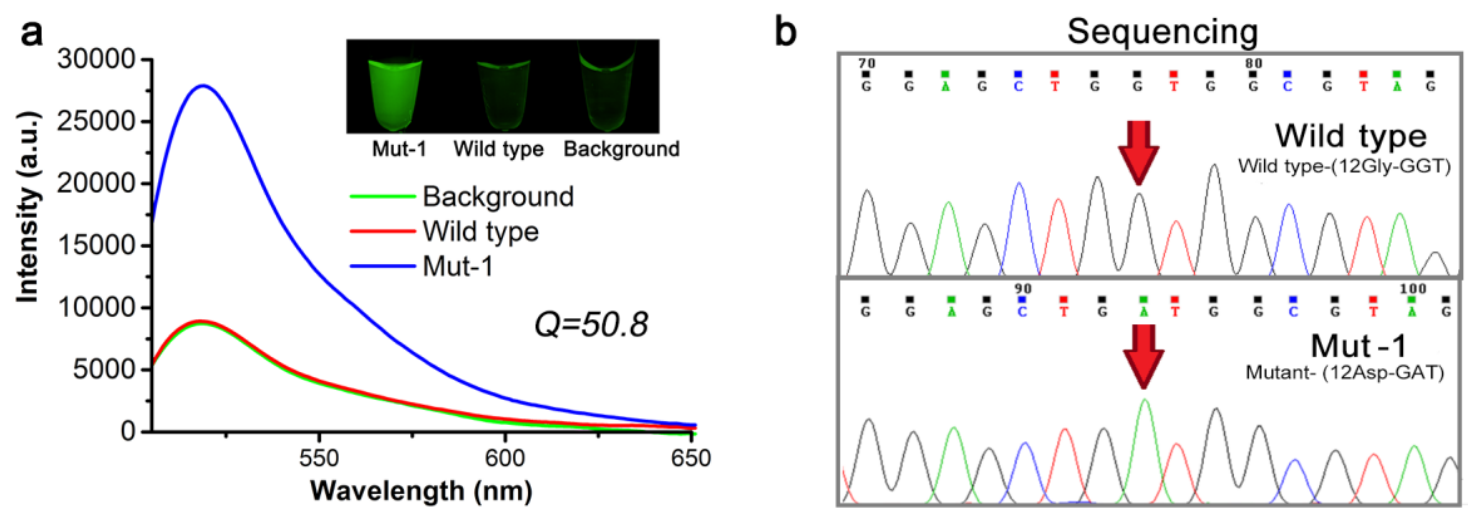

Fig. 2. Different responses of the probe to intended mutation versus wild-type sequences. (a) Comparison of the fluorescent response of the single-base mutant target and the wild-type target. The background curve represents the background fluorescence generated by the probe mixed with buffer only. The fluorescence intensities were measured by Horiba FluoroMax-4 spectrofluorometer (excitation at $493 \mathrm{~nm}$, emission at $518 \mathrm{~nm}$ ). Q was the average value of at least 3 replicates of this experiment (standard deviation=10.8). The concentration of $\mathrm{Na}^{+}$was $500 \mathrm{mM}$. The experiments were conducted at room temperature $\left(25^{\circ} \mathrm{C}\right)$. (b) Sequencing results of the targets. Wild: wild type-(12Gly-GGT). Mut-1: mutant-(12Asp-GAT). 

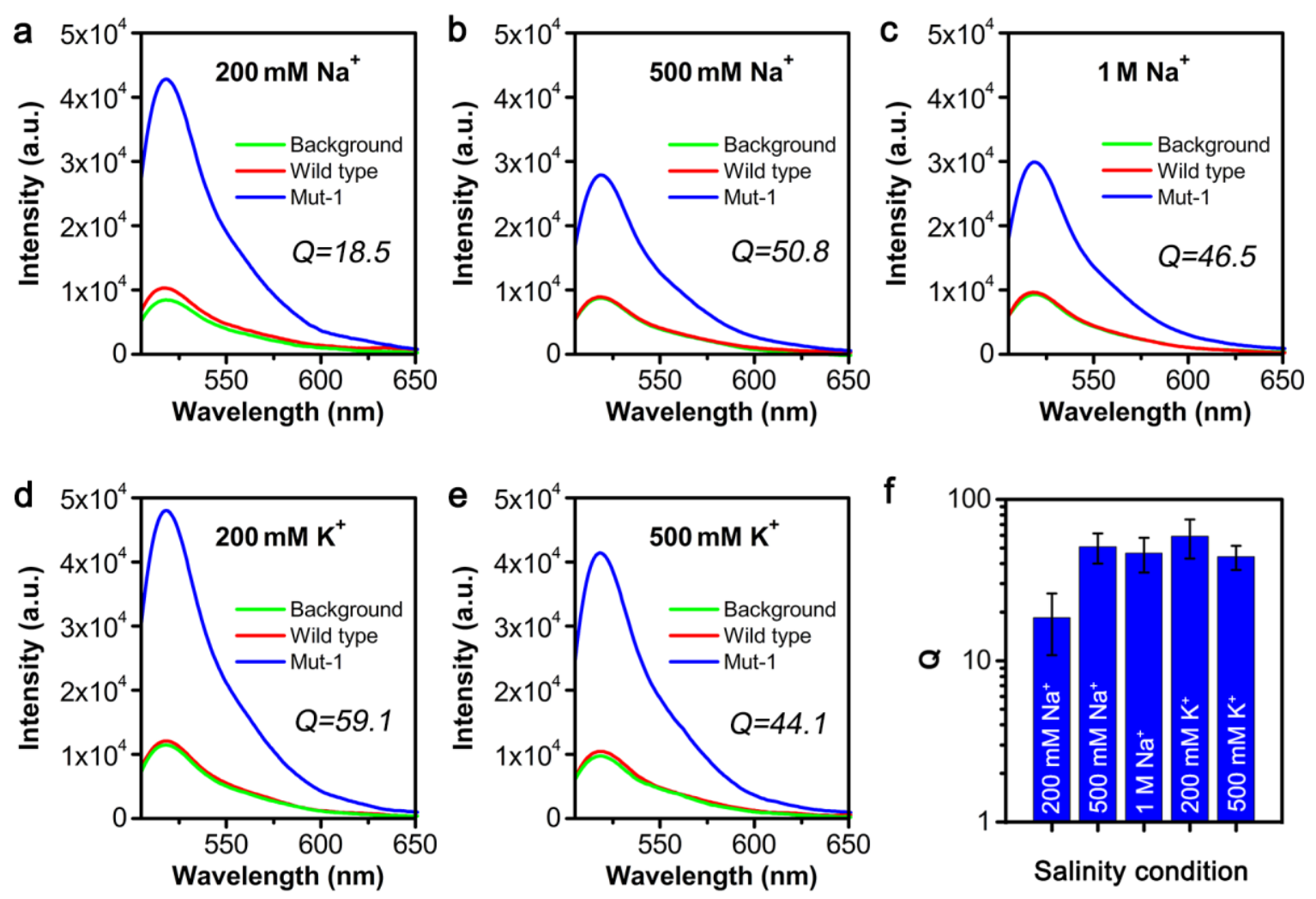

Fig. 3. The probe is capable of discriminating single-base mutations robustly in buffer containing (a) $200 \mathrm{mM} \mathrm{Na}^{+}$, (b) $500 \mathrm{mM} \mathrm{Na}^{+}$, (c) $1 \mathrm{M} \mathrm{Na}^{+}$, (d) $200 \mathrm{mM} \mathrm{K}^{+}$, and (e) $500 \mathrm{mM} \mathrm{K}^{+}$. Q was the average value of at least 3 replicates of each experiment. (f) $\mathrm{Q}$ values in different salinity conditions. Error bars are the standard deviations based on at least 3 replicates of each experiment. The emission spectra were measured by Horiba FluoroMax-4 spectrofluorometer (excitation at $493 \mathrm{~nm}$, emission at $518 \mathrm{~nm})$. The experiments were conducted at room temperature $\left(25^{\circ} \mathrm{C}\right)$. 

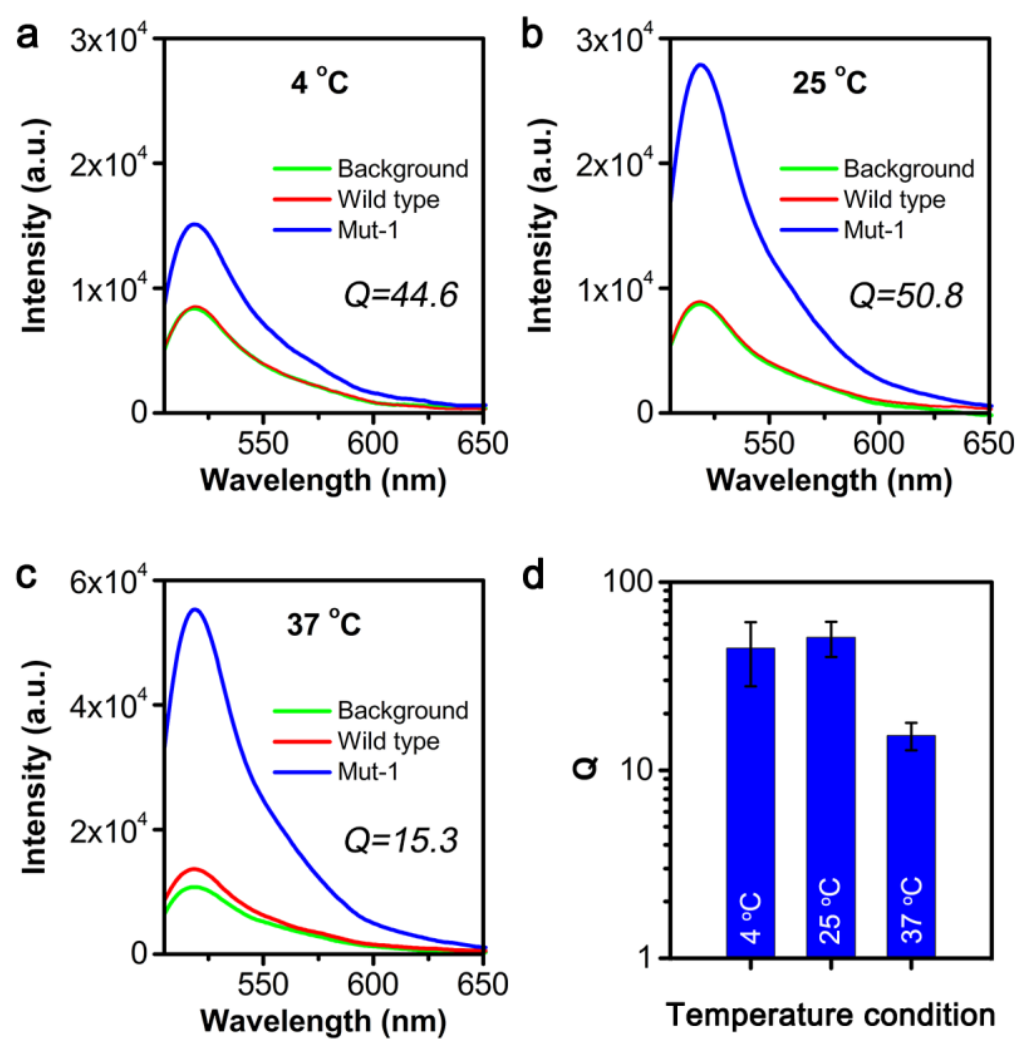

Fig. 4. The probe discriminates single-base mutations robustly at (a) $4^{\circ} \mathrm{C}$, (b) $25^{\circ} \mathrm{C}$, and (c) $37^{\circ} \mathrm{C}$. Q was the average value of at least 3 replicates of each experiment. (d) Q values in different temperature conditions. Error bars are the standard deviations based on at least 3 replicates of each experiment. The concentration of $\mathrm{Na}^{+}$was $500 \mathrm{mM}$. 

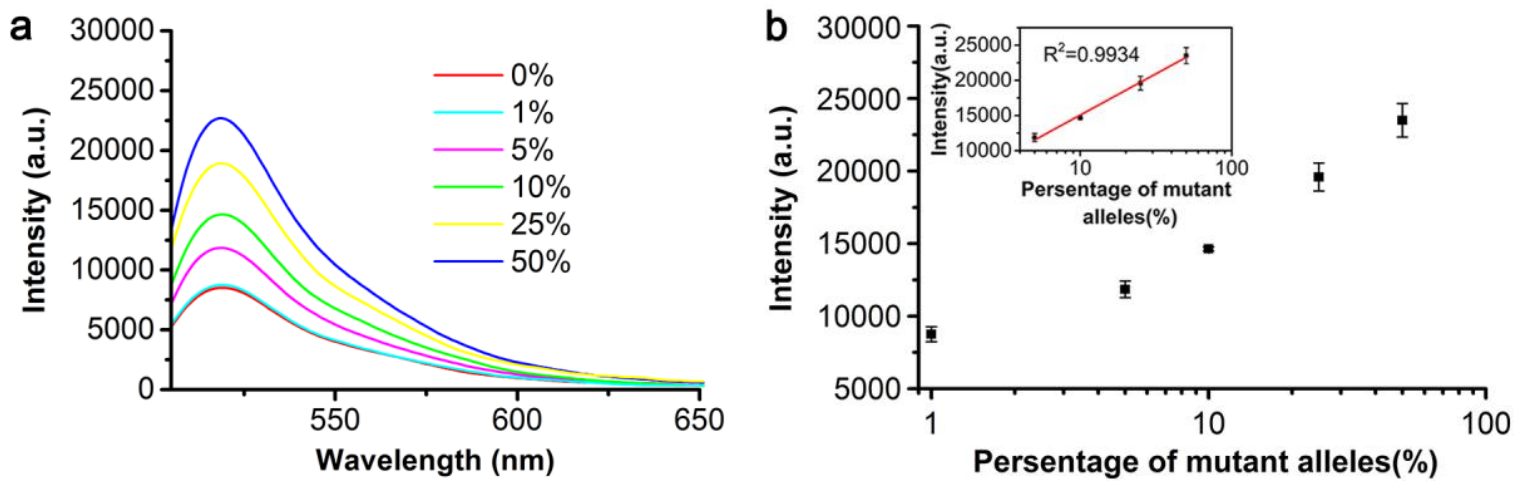

C

Sequencing
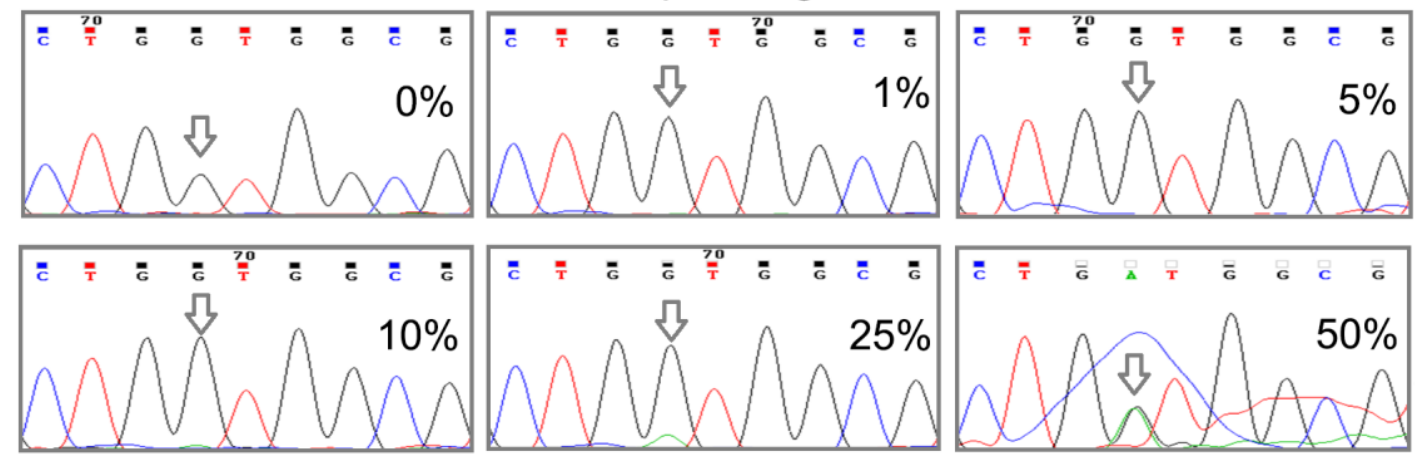

Fig. 5. Detection of KRAS mutation with serial mixtures of mutant templates and wild-type templates.

The concentration of $\mathrm{Na}^{+}$was $500 \mathrm{mM}$. The experiments were conducted at room temperature $\left(25^{\circ} \mathrm{C}\right)$. The percentages of mutant templates were $50 \%, 25 \%, 10 \%, 5 \%, 1 \%$ and $0 \%$, respectively. (a) Emission spectra of the fluorescent response to the mixed templates of different ratios. (b) Fluorescence intensity (emission at $518 \mathrm{~nm}$ ) vs. percentages of mutant templates. Error bars are the standard deviation based on at least 3 replicates of each experiment. (c) Sequencing results of the targets with different percentages of mutant templates. 
a

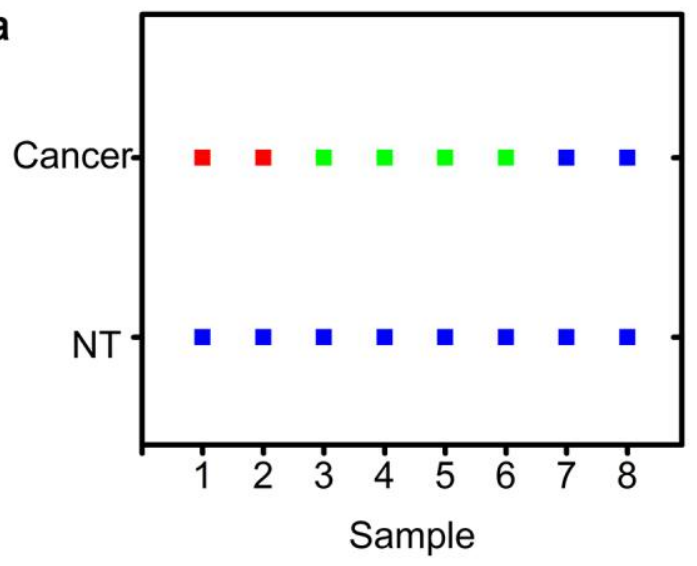

b

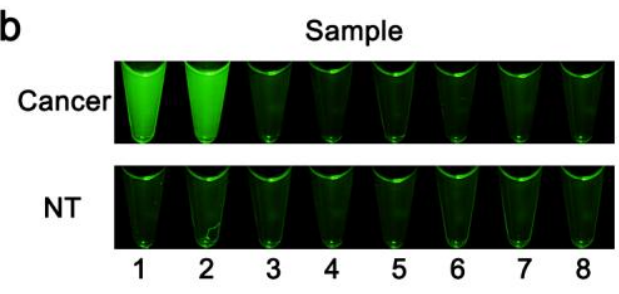

C

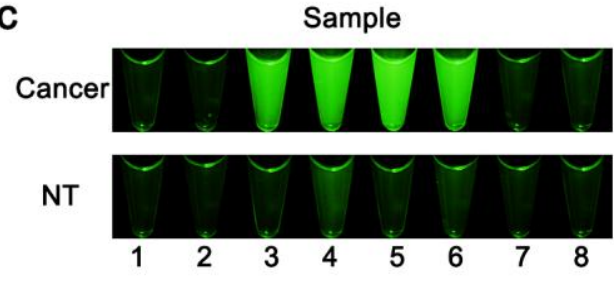

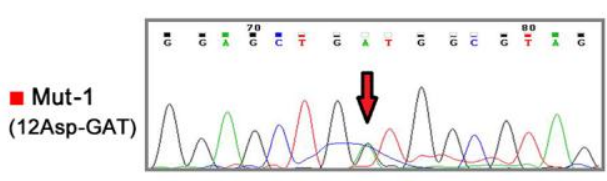
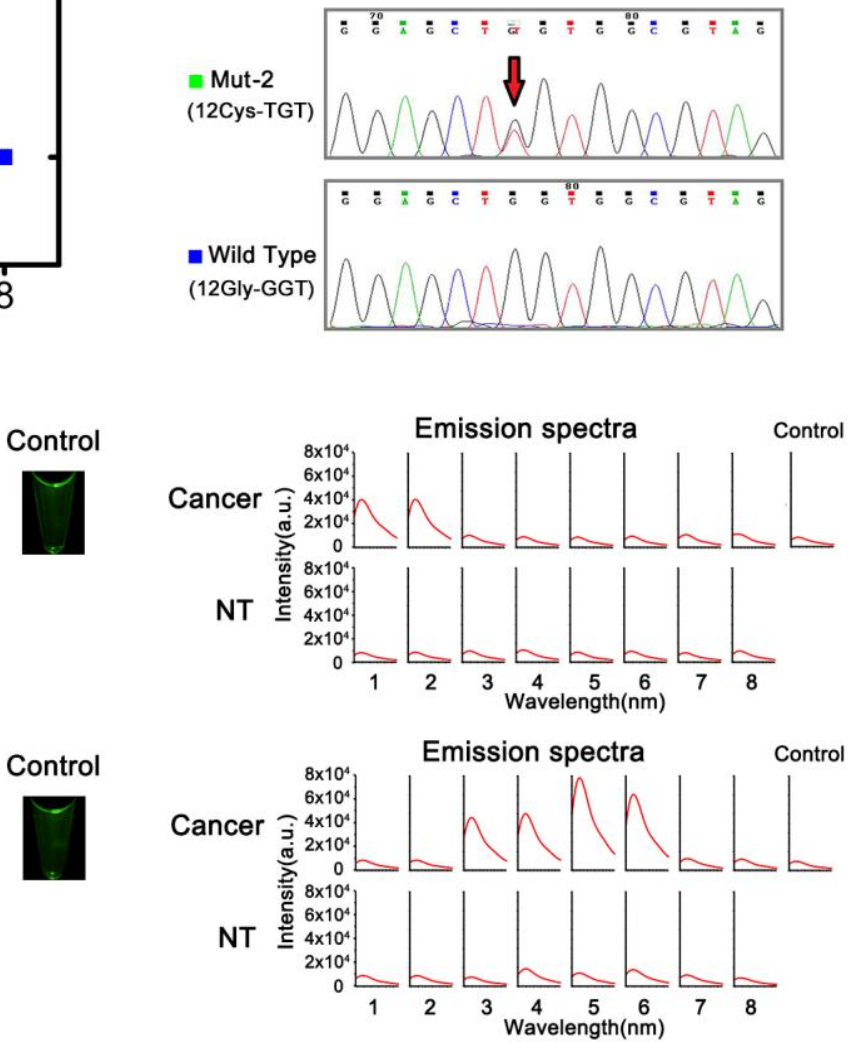

Fig. 6. (a) Sequencing results of the 8 pairs of patient cancer/NT samples. Sample 1, 2 are colorectal cancer tissues, and sample 3-8 are lung adenocarcinoma tissues. (b) Detection of the Mut-1. "Control" represents the fluorescent intensity generated by wild-type templates. (c) Detection of Mut-2. 